\title{
3 Research Square

\section{Differential Circular RNA Expression Analysis for Screening the Potential Biomarkers in Atrial Fibrillation Patients}

\section{Shenjie Sun}

Tsinghua University

Tingting LV

Beijing Tsinghua Changgung Hospital

\section{Siyuan Li}

Beijing Tsinghua Changgung Hospital

Peng Liu

Tsinghua University

\section{Ying Yang}

Tsinghua University

\section{Yuanwei Liu}

Beijing Tsinghua Changgung Hospital

Fei She

Beijing Tsinghua Changgung Hospital

\section{Rong He}

Beijing Tsinghua Changgung Hospital

Ping Zhang ( $\nabla$ zhpdoc@126.com )

Beijing Tsinghua Changgung Hospital https://orcid.org/0000-0002-5843-1108

\section{Research}

Keywords: Atrial fibrillation, circRNA, miRNA, differentially expressed genes, biomarker

Posted Date: May 4th, 2021

DOI: https://doi.org/10.21203/rs.3.rs-431598/v1

License: (c) (1) This work is licensed under a Creative Commons Attribution 4.0 International License. Read Full License 


\section{Abstract}

Background: Atrial fibrillation (AF) is the most common arrhythmia worldwide. Accumulating evidence indicated circular RNA (circRNA) as an epigenetic regulator play a critical role in the development of various of cardiovascular diseases. However, the regulatory network of circRNA/miRNA/mRNA involved in AF was still little investigated.

Methods: In the present study, AF-related differentially expressed circRNAs (DE circRNAs) were derived from the Gene Expression Omnibus (GEO) datasets GSE97455 by bioinformatic analysis. Then, miRNAs targeted by these circRNAs were predicted using the Circular RNA Interactome. These putative miRNAs were next used to predict mRNAs using three databases including miRDB, miRTarBase, and TargetScan. The targeted mRNAs were the intersection of the above-mentioned predicted mRNAs overlapping with differentially expressed genes (DEGs) identified from GEO datasets GSE79768.

Results: We firstly screened 46 DE circRNAs, 90 putative miRNAs, and 32 overlapping genes in the network of circRNA/miRNA/mRNA constructed by Cytoscape. Further, the top 7 DE circRNAs (hsa_circ_0000367, hsa_circ_0000691, hsa_circ_0000288, hsa_circ_0006168, hsa_circ_0001666, hsa_circ_0021652, and hsa_circ_0030162) were screened in the constructed circRNA/miRNA/mRNA regulatory network consisting of 7 circRNAs, 42 miRNAs, and 25 mRNAs, including 53 circRNA-miRNA pairs and 64 miRNA-mRNA pairs, which were closely related to AF.

Conclusion: Our findings revealed that $7 \mathrm{DE}$ circRNAs were involved in a circRNA/miRNA/mRNA regulatory network in AF and may be potential novel biomarkers in AF.

\section{Background}

Atrial fibrillation (AF) is the most common arrhythmia worldwide [1], and its global prevalence is between $2 \%$ and $4 \%[2,3]$. As a kind of chronic cardiac disease, AF seriously damages health by increasing the risks of stroke, heart failure, and mortality [4]. At present, rhythm or rate control and stroke prevention are major strategies for treating AF $[5,6]$. Exploring the etiology of $A F$ is crucial in searching for improved treatment.

As a stable circular transcript, circular RNA (circRNA) is prior to become a kind of potential biomarker for the diagnosis and therapy of disease[7]. One previous study reported that circRNA exacerbated cardiac hypertrophy [6], increased cardiomyocyte proliferation [8], and suppressed myocardial fibrosis [9]. Therefore, many recent studies have focused on the impact of noncoding protein RNA, especially circRNA, on the occurrence and maintenance of AF. Recent study found that two circRNA/miRNA/mRNA axes played an important role in AF according to Multi-omics research, one contained human circRNA_405917, circRNA_008132, circRNA_104052, circRNA_101021, circRNA_101020, and circRNA_102341/ miR-892b/GJA1 (Gap Junction Protein Alpha 1) and the other contained circRNA_100612/miR-133b/KCNIP1 (Potassium Voltage-Gated Channel Interacting Protein 1), JPH2 (Junctophilin 2), and ADRB1 (Adrenoceptor Beta 1)[10]. 
To clarify the potential role of circRNA/miRNA/mRNA network in AF, we adopted a more stringent adjusted $P$ value and multi-omics analysis method to explore the complex interactions of circRNA/miRNA/mRNA regulatory network in AF. Finally, we screened for differentially expressed genes (DEGs) and differentially expressed circRNAs (DE circRNAs) in patients with AF and those with a sinus rhythm (SR) from public circRNA and mRNA expression data in the Gene Expression Omnibus (GEO) database. The DE circRNAs and DEGs were identified between the groups by bioinformatic analysis. The circRNA/miRNA/mRNA regulatory network was constructed to identify the key circRNAs associated with AF that could be further explored as candidate biomarkers.

\section{Results}

\section{Differential expression analysis of circRNAs and mRNAs in AF}

13,295 probes and 10,029 circRNAs in GSE97455 were analyzed in the present study. If the $t$-test adjusted P-value was less than 0.05 and the fold change was more than 2 , further analysis using the limma package revealed 46 DE circRNAs, including 29 downregulated and 17 upregulated ones, which are shown in detail in Supplementary Table S1. The DE circRNA expression levels are presented in heat maps drawn by the limma package of R software (Fig. 1A). The DE circRNA distribution based on logFC and adjusted $P$ value is illustrated in a volcano map drawn using the pheatmap package of $R$ software (Fig. 2A). Next, we identified 488 DEGs in GSE79768, including 427 downregulated and 61 upregulated ones. Specific detailed information is shown in Supplementary Table S2. The heatmap and volcano map are shown in Fig. 1B and 2B, respectively. To better display the results, the heatmap only showed the distribution and values of DEGs, including the top 22 up-regulated genes ( $\log F C>1.5$ ) and top 47 downregulated genes $(\log \mathrm{FC}<1.5)$.

\section{PPI network and functional enrichment analysis of mRNAs in AF}

In the study, 758 nodes and 1516 edges observed in the PPI network consisting of 324 DEGs in GSE79768 using String and Cytoscape. The top 41 hub genes were identified based on cytoHubba's Degree ranking greater than 10 in Cytoscape (Supplementary Table S3). Functional enrichment analysis using R clusterProfiler package indicated $128 \mathrm{GO}$ analysis results included in the categories of biological process and cellular component in GSE79768 (Supplementary Table S4). The results of the top 20 GO analysis were illustrated in Fig. 2C, ordered by GeneRatio and P value. The top five GO terms by count were the biological process terms, including muscle system process, leukocyte migration, regulation of inflammatory response, regulation of lymphocyte activation, and positive regulation of response to external stimulus. The cellular component terms were external side of plasma membrane, collagencontaining extracellular matrix, and MHC class II protein complex. In addition, the top five KEGG terms were cytokine-cytokine receptor interaction, influenza A, hematopoietic cell lineage, leishmaniasis, and inflammatory bowel disease (Fig. 2D) (Supplementary Table S5). 


\section{Construction of circRNA/miRNA/mRNA regulatory network in $\mathrm{AF}$}

We predicted 298 miRNAs targeted by 46 DE circRNAs and obtained 1,181 circRNA-miRNA pairs using Circular RNA Interactome online tools (Supplementary Table S6-7). Subsequently, we predicted that 13,821 genes appeared in any of at least two prediction tools of miRDB, miRTarBase, and TargetScan and obtained 68,526 miRNA-mRNA pairs, including 189 putative miRNAs (Supplementary Table S8-9).

Thirty-two overlapping genes were obtained by taking the intersection between 13,821 putative genes and the top 41 hub genes in GSE79768 (Supplementary Table S10). From this we constructed the circRNA/miRNA/mRNA regulatory network including 42 circRNAs, 90 miRNAs, and 32 mRNAs forming 376 circRNA-miRNA pairs and 134 miRNA-mRNA pairs in the network (Fig. 3) (Supplementary Table S11-14).

If the fold change was greater than 4, we can get the top 7 DE circRNAs to construct the circRNA/miRNA/mRNA regulatory network. Overall, there were 7 circRNAs, 42 miRNAs, and 25 mRNAs forming 53 circRNA-miRNA pairs and 64 miRNA-mRNA pairs in the network (Fig. 4) (Supplementary Table S15-19). The 7 DE circRNAs, namely, hsa_circ_0000367, hsa_circ_0000691, hsa_circ_0000288, hsa_circ_0006168, hsa_circ_0001666, hsa_circ_0021652, and hsa_circ_0030162, were all downregulated, which were shown in Table 1. Among the 25 selected mRNAs, KRT19 (keratin 19) and IL1B (interleukin 1 beta) were up-regulated, and the remaining 23 were down-regulated in AF patients

Table 1

The chromosomal location of circRNA and genes producing every analyzed circRNA.

\begin{tabular}{|lllll|}
\hline circRNA ID & logFC & chromosomal location & best transcript & $\begin{array}{l}\text { gene producing } \\
\text { circRNA }\end{array}$ \\
\hline hsa_circ_0000367 & -3.470394456 & $\begin{array}{l}\text { chr11:124517260- } \\
124518071\end{array}$ & NM_001199922 & SIAE \\
\hline hsa_circ_0000691 & -2.415921748 & $\begin{array}{l}\text { chr16:31093273- } \\
31093358\end{array}$ & NM_014699 & ZNF646 \\
hsa_circ_0000288 & -2.195652444 & $\begin{array}{l}\text { chr11:34111725- } \\
\text { 34112225 }\end{array}$ & NM_005898 & CAPRIN1 \\
\hline hsa_circ_0006168 & -2.154812501 & $\begin{array}{l}\text { chr4:78694234- } \\
78697546\end{array}$ & NM_144571 & CNOT6L \\
\hline hsa_circ_0001666 & -2.111649959 & $\begin{array}{l}\text { chr6:170626457- } \\
170639638\end{array}$ & NM_032448 & FAM120B \\
\hline hsa_circ_0021652 & -2.01480741 & $\begin{array}{l}\text { chr11:34110941- } \\
\text { 34112225 }\end{array}$ & NM_005898 & CAPRIN1 \\
hsa_circ_0030162 & -2.012708424 & $\begin{array}{l}\text { chr13:45911303- } \\
\text { 45914319 }\end{array}$ & NM_003295 & TPT1 \\
\hline
\end{tabular}


The GO and KEGG functional enrichment analysis results were used to predict the potential functions using the circRNA/miRNA/mRNA regulatory network in AF. In the potential AF-associated signaling pathway, GO analysis showed that the calcium ion transport-related pathway and the second-messengermediated signaling pathway contained the hub genes ADRA2A (adrenoceptor alpha 2A), CXCL11 (C-X-C motif chemokine ligand 11), P2RY12 (purinergic receptor P2Y12), and PTGS2 (prostaglandinendoperoxide synthase 2); the programmed cell death pathway contained the hub genes IL1B, KIT (tyrosine-protein kinase Kit), and KITLG (KIT ligand); and the renin-angiotensin pathway included AGTR2 (angiotensin II receptor type 2). The potential relationship among the hub genes, the circRNA/miRNA/mRNA regulatory network, and functional enrichment analysis in AF (Supplementary Table S20).

\section{Discussion}

In recent years, although cardiologists have made great progress for the diagnosis and treatment of AF, the high incidence and high disability rate induced by AF still constitute a heavy medical burden globally. And some points regarding the pathogenesis of AF are still unclear. In this study, we constructed a regulatory network of 7 circRNAs, 42 miRNAs, and 25 mRNAs using GEO datasets to explore the potential circRNA involved in AF. Combining the results of enrichment analysis, we determined the occurrence mechanism in calcium signaling-related pathway, second messenger-mediated signaling-related pathway, programmed cell death pathway, and renin-angiotensin pathway (Supplementary Table S20).

There are four hub genes, namely, ADRA2A, P2RY12, CXCL11, and PTGS2, involved in the regulation of calcium ion transport pathway and second messenger-mediated signaling pathway. The hsa_circ_0001666/hsa_miR-1827/ADRA2A pathway involved in AF through the second messenger signaling pathway. Previous study also showed that lacking ADRA2A elevated plasma noradrenaline concentrations and participated in the development of cardiac hypertrophy and dysfunction[11]. In addition, hsa_circ_0000288, hsa_circ_0021652/hsa_miR-548m/P2RY12 network also played an important role in $A F$ through a similar mechanism. The latest research illustrated that the crosstalk between P2Y12 and IGF-I receptors can increase PKB phosphorylation through calcium-dependent activation of the Pyk2/Src pathway[12]. The hub gene PTGS2 involved in more complex circRNA/miRNA/mRNA regulatory networks in AF including 5 circRNA, such as hsa_circ_0001666, hsa_circ_0006168, hsa_circ_0021652, hsa_circ_0000288, and hsa_circ_0030162. Previous research also demonstrated that the combination of PTGS2 and miRNA-26 can inhibit the MAPK pathway and reduce the inflammatory response and myocardial remodeling of mice with myocardial infarction[13].

Moreover, there are three hub genes, namely, IL1B, KIT, and KITLG, involved in the programmed cell death pathway. Our research data revealed that the hsa_circ_0001666/hsa_miR-590-5p/IL1B involved in AF via programmed cell death pathway. And hsa_circ_0021652, hsa_circ_0000288/hsa_miR-583/KIT networks also participated in AF via similar pathway. Previous research results suggested that IL1B genetic variation is involved in mediating CRP expression [14]. In reviewing the literature, no data was found about the association between CXCL11, KIT, and KITLG in cardiac diseases. The hsa_circ_0001666/miR- 
1827/ADRA2A pathway, the hsa_circ_0000288 or hsa_circ_0021652/miR-548m/P2RY12 pathway, circ_0001666/miR-590-5p/IL1B pathway, will become the target regulation network of our research.

Furthermore, a total of four miRNAs, namely, miR-1263, miR-1299, miR-1322, and miR-330-3p, were involved in the renin-angiotensin system and second messenger-mediated signaling pathway with the AGTR2 gene. Previous studies revealed that AGTR2 is involved in regulating collagen accumulation and MMP expression[15]. The network showed that hsa_circ_0001666 can adsorb three miRNAs, namely, miR1299, miR-1322, and miR-330-3p, while hsa_circ_0000367 interacts with miR-1263. The other three circRNAs, hsa_circ_0030162, hsa_circ_0021652, and hsa_circ_0000288, can also interact with miR-1322 to influence AGTR2 expression in AF. The regulatory network involved in AGTR2 can become the most valuable target in our future research work.

The main limitation of this study is the small sample size, which may have resulted in selection bias. Therefore, further genomics research on more specimen sources is required to validate these results. We are currently preparing to perform knockdown and overexpression experiments to explore the effects of changes in the expression of circRNAs and associated key genes using cell and animal models. Despite these limitations, the findings of this study suggest that circRNAs are also potential biomarkers or therapeutic targets for $\mathrm{AF}$, offering a guide for further research to better understand the mechanism of $A F$.

\section{Conclusions}

In conclusion, we constructed a circRNA/miRNA/mRNA regulatory network including seven highly expressed circRNAs (hsa_circ_0000367, hsa_circ_0000691, hsa_circ_0000288, hsa_circ_0006168, hsa_circ_0001666, hsa_circ_0021652, and hsa_circ_0030162) through GEO datasets and performed GO and KEGG analyses to explore the potential mechanisms of action of circRNAs in AF. These circRNAs are worthy of further exploration for use as novel biomarkers in AF.

\section{Materials And Methods \\ Data Retrieval}

We downloaded original files on circRNA and mRNA expression from the GEO database (http://www.ncbi.nlm.nih.gov/geo/). The circRNA expression data were derived from the GSE97455 datasets, including 15 pairs of plasma specimens of AF and a control group. The GPL21825 platforms were generated using Arraystar Human CircRNA microarray V2. The mRNA expression data were obtained from GSE79768 datasets including seven left atrial specimens with AF and six specimens with SR. The miRNAs targeted by circRNAs were predicted using Circular RNA Interactome online tools (https://circinteractome.nia.nih.gov/) [16].

\section{Differential expression analysis of circRNAs and mRNAs in AF}


The probe name was converted to the circRNA international standard symbol by using the GPL21825 annotation file in the GSE97455 series matrix with Perl software 5.30.1. Differentially expressed circular RNAs (DE circRNAs) were analyzed using the limma package of $R$ software. DE circRNAs were defined based on the adjusted p-value was less than 0.05 and the fold change was more than 2 . The CEL files of GSE79768 were cleaned including background correction, data normalization, and expression level calculation to form a gene expression level matrix. We converted the probe name to the gene symbol using the GPL570 platform annotation file by R software. DEGs were identified according to the adjusted p-value was less than 0.05 and the fold change was more than 2 using the limma package. The missing values were filled with the weighted k-nearest-neighbor technique [17]. The $P$ value was adjusted by the Benjamini-Hochberg method.

\section{PPI and functional enrichment analysis of mRNAs in AF}

We constructed the protein-protein interaction (PPI) network to obtain the interactions of the identified DEGs in the GSE79768 by multiple protein function tools in the STRING database (STRING database, Version 11, https://string-db.org) [18]. The confidence limit of the interaction score median was set to 0.40. Cytoscape 3.7.2 was used to draw the network of the DEGs and the cytoHubba plugin components were to identify the hub genes in the PPI network sorted by the degree ranking table (https://cytoscape.org/) [19]. We performed functional enrichment analysis on DEGs in GSE79768 by use of the functional annotation tool DAVID 6.8 (https://david.ncifcrf.gov/) [20]and the R clusterProfiler package.

\section{Construction of the circRNA/miRNA/mRNA regulatory network in AF}

We predicted miRNAs and established circRNA-miRNA pairs using the DE circRNAs in Circular RNA Interactome. Putative miRNAs targeted by DE circRNAs were used to predict mRNAs by utilizing miRDB [21], miRTarBase [22] and TargetScan[23]. The intersection of predicted mRNAs and above-mentioned hub genes from GSE79768 were used to establish miRNA-mRNA pairs. Then, the circRNA/miRNA/mRNA regulatory network was constructed using Cytoscape 3.7.2.

\section{Declarations}

\section{Ethics approval and consent to participate}

Not necessary. The raw data of this study are derived from the GEO data portal, which are publicly available databases.

\section{Consent for publication}

Not applicable. 


\section{Competing interests}

The authors declare that they have no competing interests.

\section{Funding}

This project is supported by Beijing Municipal Administration of Hospitals Clinical medicine Development of special funding support (ZYLX201813) and Beijing Municipal Administration of Hospitals' Ascent Plan (DFL20190902). The funders had no role in study design, data collection and analysis, decision to publish, or preparation of the manuscript.

\section{Authors' contributions}

(I) Conception and design: Shenjie Sun; (II) Administrative support: Ping Zhang; (III) Provision of study materials: All authors; (IV) Collection and assembly of data: All authors; (V) Data analysis and interpretation: All authors; (VI) Manuscript writing: All authors; (VII) Final approval of manuscript: All authors.

\section{Acknowledgements}

The authors gratefully acknowledge the Gene Expression Omnibus (GEO) database, which made the data available. We acknowledge GEO database for providing their platforms and contributors for uploading their meaningful datasets.

\section{Availability of data and materials}

The raw data of this study are derived from the GEO data portal (https:// www. ncbi. nlm. nih. gov/ geo/), which are publicly available databases.

\section{References}

1. Chugh SS, Havmoeller R, Narayanan K, Singh D, Rienstra M, Benjamin EJ, Gillum RF, Kim YH, McAnulty JH Jr, Zheng ZJ, et al. Worldwide epidemiology of atrial fibrillation: a Global Burden of Disease 2010 Study. Circulation. 2014;129(8):837-47.

2. Benjamin EJ, Muntner P, Alonso A, Bittencourt MS, Callaway CW, Carson AP, Chamberlain AM, Chang AR, Cheng S, Das SR, et al. Heart Disease and Stroke Statistics-2019 Update: A Report From the American Heart Association. Circulation. 2019;139(10):e56-28.

3. Hindricks G, Potpara T, Dagres N, Arbelo E, Bax JJ, Blomstrom-Lundqvist C, Boriani G, Castella M, Dan GA, Dilaveris PE, et al. 2020 ESC Guidelines for the diagnosis and management of atrial fibrillation developed in collaboration with the European Association for Cardio-Thoracic Surgery (EACTS). Eur Heart J. 2021;42(5):373-498.

4. Ruddox V, Sandven I, Munkhaugen J, Skattebu J, Edvardsen T, Otterstad JE. Atrial fibrillation and the risk for myocardial infarction, all-cause mortality and heart failure: A systematic review and meta- 
analysis. Eur J Prev Cardiol. 2017;24(14):1555-66.

5. Kirchhof P, Benussi S, Kotecha D, Ahlsson A, Atar D, Casadei B, Castella M, Diener HC, Heidbuchel H, Hendriks J, et al. 2016 ESC Guidelines for the management of atrial fibrillation developed in collaboration with EACTS. Eur Heart J. 2016;37(38):2893-962.

6. January CT, Wann LS, Calkins H, Chen LY, Cigarroa JE, Cleveland JC Jr, Ellinor PT, Ezekowitz MD, Field ME, Furie KL, et al. 2019 AHA/ACC/HRS Focused Update of the 2014 AHA/ACC/HRS Guideline for the Management of Patients With Atrial Fibrillation: A Report of the American College of Cardiology/American Heart Association Task Force on Clinical Practice Guidelines and the Heart Rhythm Society in Collaboration With the Society of Thoracic Surgeons. Circulation. 2019;140(2):e125-51.

7. Rybak-Wolf A, Stottmeister C, Glazar P, Jens M, Pino N, Giusti S, Hanan M, Behm M, Bartok O, AshwalFluss R, et al. Circular RNAs in the Mammalian Brain Are Highly Abundant, Conserved, and Dynamically Expressed. Mol Cell. 2015;58(5):870-85.

8. Huang S, Li X, Zheng H, Si X, Li B, Wei G, Li C, Chen Y, Chen Y, Liao W, et al. Loss of Super-EnhancerRegulated circRNA Nfix Induces Cardiac Regeneration After Myocardial Infarction in Adult Mice. Circulation. 2019;139(25):2857-76.

9. Zhou B, Yu JW. A novel identified circular RNA, circRNA_010567, promotes myocardial fibrosis via suppressing miR-141 by targeting TGF-beta1. Biochem Biophys Res Commun. 2017;487(4):769-75.

10. Jiang S, Guo C, Zhang W, Che W, Zhang J, Zhuang S, Wang Y, Zhang Y, Liu B. The Integrative Regulatory Network of circRNA, microRNA, and mRNA in Atrial Fibrillation. Front Genet. 2019;10:526.

11. Hein L, Altman JD, Kobilka BK. Two functionally distinct alpha2-adrenergic receptors regulate sympathetic neurotransmission. Nature. 1999;402(6758):181-4.

12. Van Kolen K, Gilany K, Moens L, Esmans EL, Slegers H. P2Y12 receptor signalling towards PKB proceeds through IGF-I receptor cross-talk and requires activation of Src, Pyk2 and Rap1. Cell Signal. 2006;18(8):1169-81.

13. Ge ZW, Zhu XL, Wang BC, Hu JL, Sun JJ, Wang S, Chen XJ, Meng SP, Liu L, Cheng ZY. MicroRNA-26b relieves inflammatory response and myocardial remodeling of mice with myocardial infarction by suppression of MAPK pathway through binding to PTGS2. Int J Cardiol. 2019;280:152-9.

14. Enquobahrie DA, Rice K, Williams OD, Williams MA, Gross MD, Lewis CE, Schwartz SM, Siscovick DS. IL1B genetic variation and plasma C-reactive protein level among young adults: the CARDIA study. Atherosclerosis. 2009;202(2):513-20.

15. Dandapat A, Hu CP, Chen J, Liu Y, Khan JA, Remeo F, Carey RM, Hermonat PL, Mehta JL. Overexpression of angiotensin II type 2 receptor (agtr2) decreases collagen accumulation in atherosclerotic plaque. Biochem Biophys Res Commun. 2008;366(4):871-7.

16. Dudekula DB, Panda AC, Grammatikakis I, De S, Abdelmohsen K, Gorospe M. Circlnteractome: A web tool for exploring circular RNAs and their interacting proteins and microRNAs. RNA Biol. 2016;13(1):34-42. 
17. Troyanskaya O, Cantor M, Sherlock G, Brown P, Hastie T, Tibshirani R, Botstein D, Altman RB. Missing value estimation methods for DNA microarrays. Bioinformatics. 2001;17(6):520-5.

18. Szklarczyk D, Gable AL, Lyon D, Junge A, Wyder S, Huerta-Cepas J, Simonovic M, Doncheva NT, Morris JH, Bork P, et al. STRING v11: protein-protein association networks with increased coverage, supporting functional discovery in genome-wide experimental datasets. Nucleic Acids Res. 2019;47(D1):D607-13.

19. Shannon P, Markiel A, Ozier O, Baliga NS, Wang JT, Ramage D, Amin N, Schwikowski B, Ideker T. Cytoscape: a software environment for integrated models of biomolecular interaction networks. Genome Res. 2003;13(11):2498-504.

20. Huang da W, Sherman BT, Lempicki RA. Systematic and integrative analysis of large gene lists using DAVID bioinformatics resources. Nat Protoc. 2009;4(1):44-57.

21. Chen $Y$, Wang $X$. miRDB: an online database for prediction of functional microRNA targets. Nucleic Acids Res. 2020;48(D1):D127-31.

22. Hsu SD, Lin FM, Wu WY, Liang C, Huang WC, Chan WL, Tsai WT, Chen GZ, Lee CJ, Chiu CM, et al. miRTarBase: a database curates experimentally validated microRNA-target interactions. Nucleic Acids Res. 2011;39(Database issue):D163-9.

23. Garcia DM, Baek D, Shin C, Bell GW, Grimson A, Bartel DP. Weak seed-pairing stability and high targetsite abundance decrease the proficiency of Isy- 6 and other microRNAs. Nat Struct Mol Biol. 2011;18(10):1139-46.

\section{Figures}



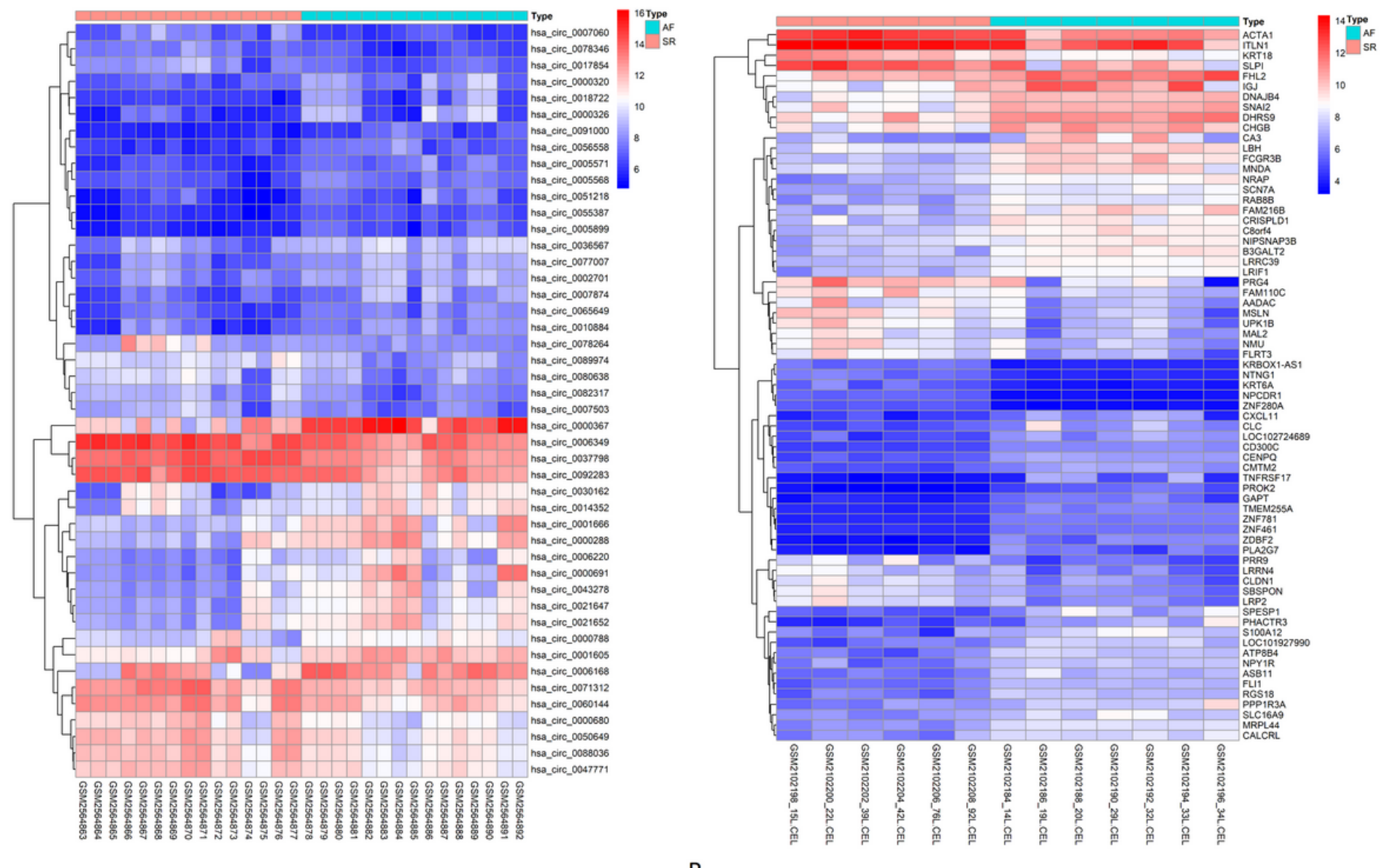

A

B

\section{Figure 1}

Heat map of DE circRNAs in GSE97455 and DEGs in GSE79768 A. Heat map of DE circRNAs Heat map clustering of the differentially expressed circRNAs between atrial fibrillation (AF) and sinus rhythm (SR) in GSE97455 are shown. Red indicates higher expression and blue indicates lower expression. B. Heat map of DEGs in GSE79768. Heat map clustering of the differentially expressed genes between AF and SR tissue samples; only the top 47 down-regulated genes and top 22 up-regulated genes in GSE79768 are shown. Red indicates higher expression and blue indicates lower expression. 


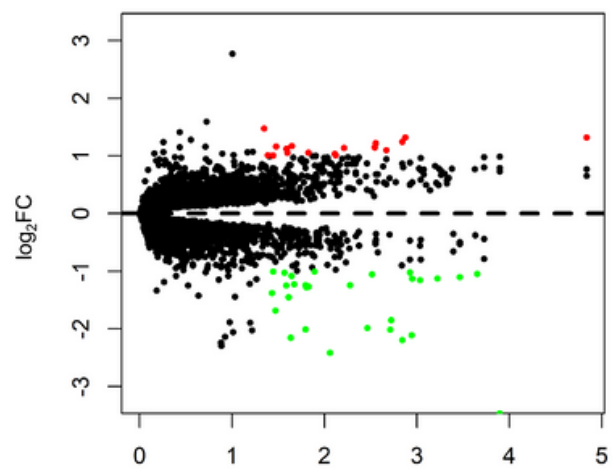

A

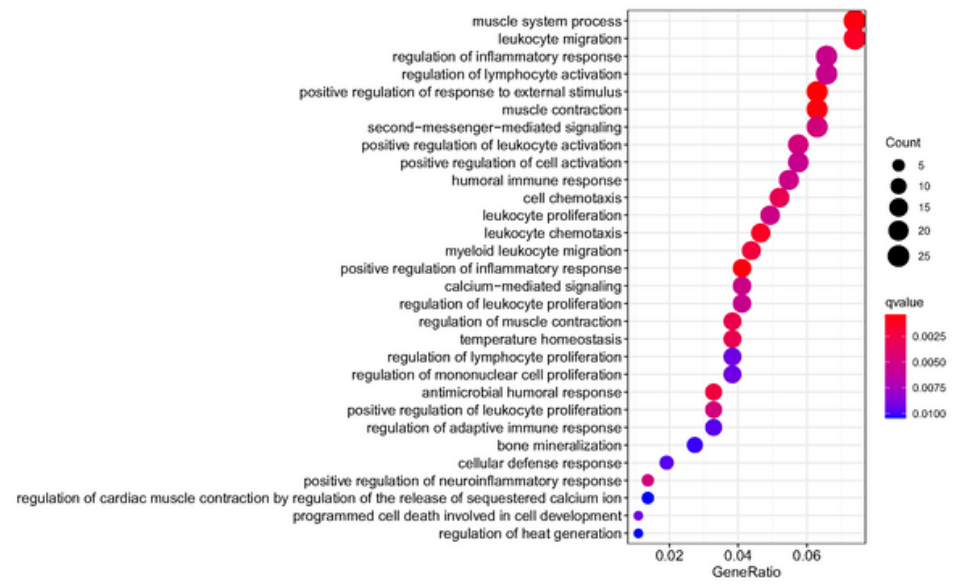

C

\section{Figure 2}

Volcano plot showing DE circRNAs and DEGs, and GO terms and KEGG pathway enrichment of all DEGs. A. Volcano plot showing DE circRNAs in AF Distribution of the differentially expressed circRNAs between atrial fibrillation (AF) and sinus rhythm (SR) samples in GSE97455 shown as a volcano map (adjusted Pvalue according to the logFC values). The red dots above the line are up-regulated circRNAs and the green dots below the line are down-regulated circRNAs, respectively. B. Volcano plot showing DEGs in AF Distribution of the differentially expressed genes between atrial fibrillation (AF) and sinus rhythm (SR) samples in GSE79768 shown as a volcano map (adjusted P-value according to the logFC values). The red dots above the line are up-regulated genes and the green dots below the line are down-regulated genes, respectively. C. GO terms enrichment GO analysis of all differentially expressed genes in GSE79768, All DEGs were classified by GO terms based on their cellular component, molecular function, and biological process. D. KEGG pathway enrichment KEGG enrichment analysis of all DEGs in GSE79768. 


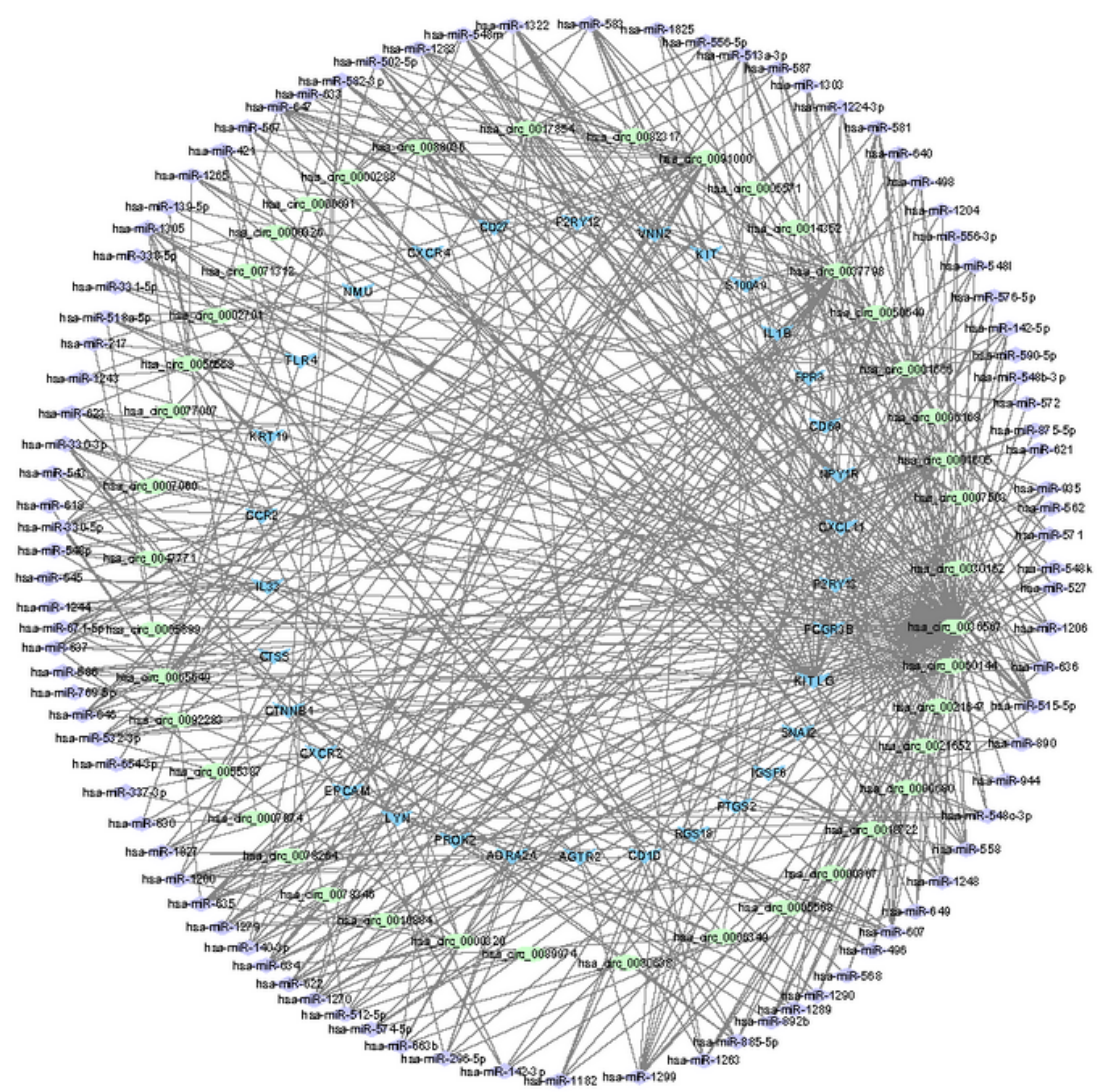

Figure 3

The circRNA / miRNA / mRNA regulatory network including 42 circRNAs The circRNA / miRNA / mRNA regulatory network including 42 circRNAs, 90 miRNAs, and 32 mRNAs forming 376 circRNA-miRNA pairs and 134 miRNA-mRNA pairs. 


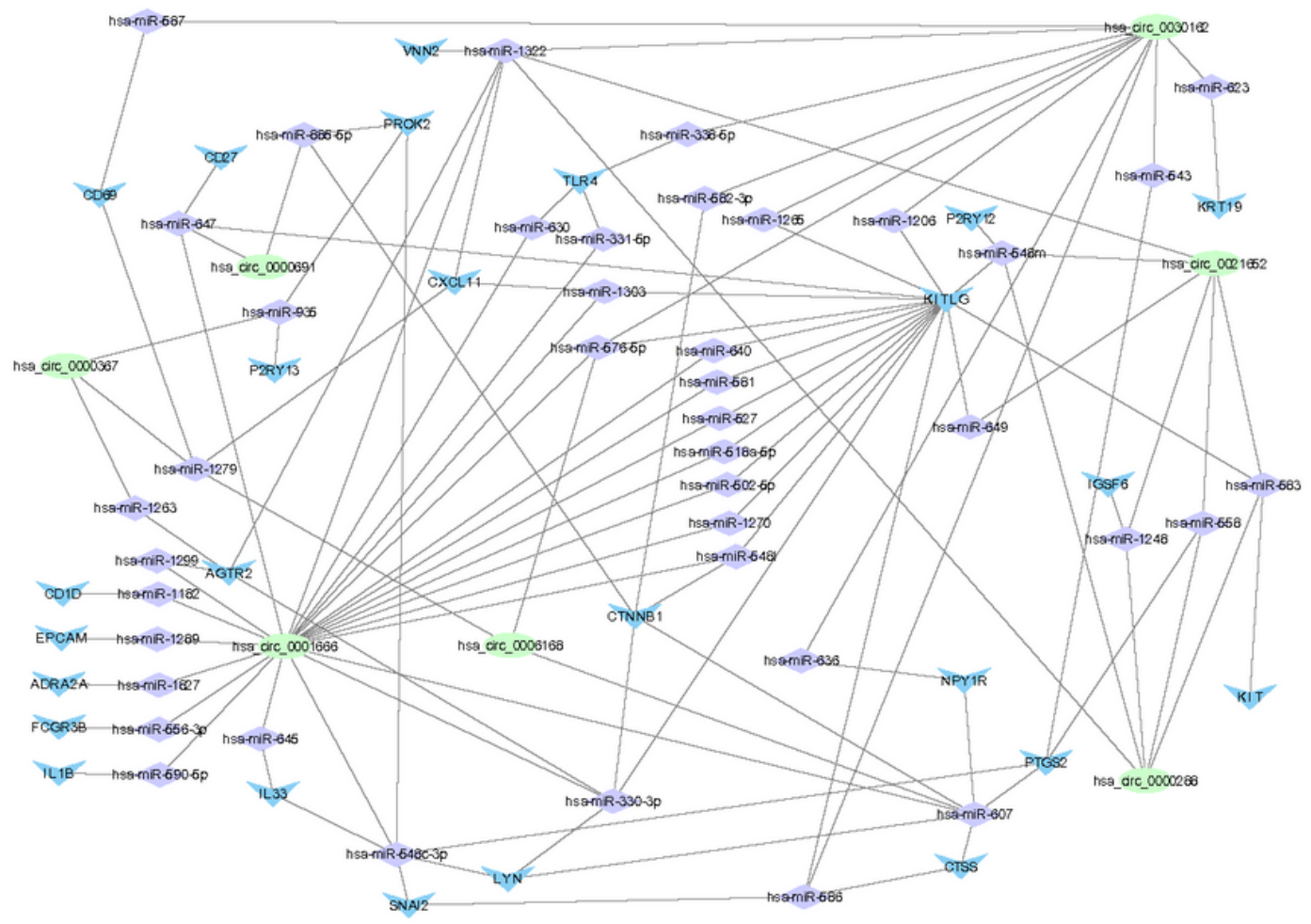

Figure 4

The circRNA / miRNA / mRNA regulatory network including 7 circRNAs. The circRNA / miRNA / mRNA regulatory network including 7 circRNAs, 42 miRNAs, and 25 mRNAs forming 53 circRNA-miRNA pairs and 64 miRNA-mRNA pairs.

\section{Supplementary Files}

This is a list of supplementary files associated with this preprint. Click to download.

- SupplementaryMaterial.docx 